\title{
Safety and Efficacy of Low-dose Pioglitazone (7.5 mg/day) vs. Standard-dose Pioglitazone (15 mg/day) in Japanese Women with Type 2 Diabetes Mellitus
}

\author{
TAKAFUMI MAJIMA*,**, YASATO KOMATSU**, KENTARO DOI*, MICHIKA SHIGEMOTO*, \\ CHIEKO TAKAGI*, ATSUSHI FUKAO***, JERRY CORNERS ${ }^{\#}$ AND KAZUWA NAKAO** \\ *Department of Endocrinology and Metabolism, Rakuwakai Otowa Hospital, Kyoto 607-8062, Japan \\ **Department of Medicine and Clinical Science, Kyoto University Graduate School of Medicine, Kyoto 606-8507, Japan \\ ***Department of Psychosomatic Medicine, Rakuwakai Otowa Hospital, Kyoto 607-8062, Japan \\ \#Medical Adviser, Rakuwakai Otowa Hospital, Kyoto 607-8062, Japan
}

\begin{abstract}
It is well known that pioglitazone, a potent thiazolidinedione, improves metabolic control. However, weight gain or peripheral edema may be of major clinical concern when using this agent. The purpose of our study was to prospectively evaluate the effects of low-dose pioglitazone $(7.5 \mathrm{mg} / \mathrm{day})$ on metabolic control, weight gain and the incidence of edema compared with a standard dose of pioglitazone $(15.0 \mathrm{mg} / \mathrm{day})$ in patients with type 2 diabetes mellitus (T2DM). Ninety-five Japanese female patients (mean age 58.4 \pm 10.4 years) with newly diagnosed T2DM were selected for this study. They were randomly divided into the following 2 groups according to therapy regimens, and examined every month for 6 months after diagnosis. Group A consisted of 54 patients treated with low-dose pioglitazone orally; Group B, the control-group, consisted of 41 patients treated with standard-dose pioglitazone orally. The incidence of peripheral edema was significantly much lower in group A $(2 / 54)$ than in group B $(11 / 41)(p=0.0014)$. In addition, $\%$ change of body weight during the 6-month treatment in group A was significantly less than that in group B ( $\mathrm{p}<0.0001)$. On the other hand, the \% change of biochemical parameters including HbA1c did not differ significantly between group A and group B, although glucose and lipid control significantly improved from baseline in both groups. Our results demonstrate the safety and efficacy of low-dose pioglitazone, suggesting that it could be another good choice of treatment for Japanese women with T2DM.
\end{abstract}

Key words: Pioglitazone, Thiazolidinedione, Diabetes mellitus

(Endocrine Journal 53: 325-330, 2006)

IT is well known that pioglitazone, a member of the thiazolidinedione (TZD) class, improves glycemic control, reduces hyperinsulinemia and improves lipid metabolism, mainly by activating peroxisome proliferatoractivated receptor- $\gamma$ (PPAR- $\gamma$ ) [1-5]. Moreover, because the antiatherogenic effects of TZDs are likely to be mediated through their direct action on the vasculature, pioglitazone may confer benefits beyond decreases

Received: May 23, 2005

Accepted: January 23, 2006

Correspondence to: Takafumi MAJIMA, M.D., Department of Endocrinology and Metabolism, Rakuwakai Otowa Hospital, 2 Otowa Chinji-cho, Yamashina-ku, Kyoto 607-8062, Japan in glucose level [1-7]. However, certain clinical concerns make clinicians hesitant to use it in Japan. One of such concerns is liver toxicity, since troglitazone, the first agent in this class to become available for clinical use in Japan, was withdrawn due to reports of severe hepatic injury [2-5]. However, we should not be overly concerned about the hepatotoxicity of pioglitazone, provided that liver function tests are performed at baseline and periodically thereafter [2-6]. Indeed, there have been a few reports of hepatotoxicity potentially caused by pioglitazone $[4,8]$. In these reports, however, the agent was never proved to be the cause of the hepatotoxicity, and the condition was resolved with supportive care and withdrawal of the putative agent $[4,8]$. 
On the other hand, fluid retention, weight gain, or peripheral edema may be of major clinical concern, because these are the most frequent adverse events associated with pioglitazone [2-6,9], occurring more often in women than in men [6]. The incidence of edema is reported to be about $5-10 \%$, although the incidence rises to $13-16 \%$ when combined with insulin therapy [4, 6, 9-15]. Fluid retention is also reported to account for a $6 \%-7 \%$ increase in plasma volume $[4,9]$; thus pioglitazone is contraindicated in patients with severe congestive heart failure (CHF), defined as New York Heart Association (NYHA) functional class III and IV $[2-5,9]$. Weight gain partly due to fluid retention is reported to be significant and dose-dependent (ranging $0.5-4.5 \mathrm{~kg})[4,10,14,15]$. These frequent and undesirable effects of pioglitazone, even if not fatal, may lead to the breakdown of the doctor-patient relationship and consequently discontinuation of treatment of type 2 diabetes mellitus (T2DM) itself. In order to reduce these adverse effects of pioglitazone, we tried a lower dosage on patients with T2DM.

The aim of our study was to prospectively evaluate the effects of low-dose pioglitazone $(7.5 \mathrm{mg} /$ day $)$ on glucose and lipid metabolism, weight gain and the incidence of edema compared with a standard-dose of pioglitazone $(15.0 \mathrm{mg} /$ day $)$ in Japanese women with T2DM.

\section{Materials and Methods}

\section{Subjects}

Ninety-five Japanese female patients (mean age $58.4 \pm 10.4$ years) with newly diagnosed T2DM who initially attended the clinic of Rakuwakai Otowa Hospital during the period between November 2003 and October 2004, were selected for this study. The diagnosis of T2DM was established on the basis of abnormal glucose tolerance test results, classic symptoms, and laboratory findings. The patients were randomly divided into the following 2 groups according to therapy regimen, and examined every month for 6 months after diagnosis. Group A consisted of 54 patients treated with low-dose pioglitazone $(7.5 \mathrm{mg} /$ day) orally; Group B, the control-group, consisted of 41 patients treated with standard-dose pioglitazone $(15.0 \mathrm{mg} /$ day $)$ orally. Their clinical data are shown in Table 1.

All subjects were instructed to adhere to a diseaseand weight-oriented diet and exercise regimen throughout the study. Dietary and exercise advice was given at baseline and every month after the diagnosis during the 6-month treatment, with the target of body weight normalization and supply of individually appropriate calories and nutrients. All subjects were examined by the

Table 1. Mean \pm SD of the variables assessed in the examined subjects

\begin{tabular}{|c|c|c|c|c|}
\hline & \multicolumn{2}{|c|}{ Group A $(\mathrm{n}=53)($ pioglitazone $7.5 \mathrm{mg} /$ day $)$} & \multicolumn{2}{|c|}{ Group B $(\mathrm{n}=31)($ pioglitazone $15 \mathrm{mg} /$ day $)$} \\
\hline & Baseline & 6 months later & Baseline & 6 months later \\
\hline Age (years) & $57.85 \pm 10.8$ & $58.29 \pm 10.9$ & $59.13 \pm 10.0$ & $59.51 \pm 10.1$ \\
\hline BMI $\left(\mathrm{kg} / \mathrm{m}^{2}\right)$ & $23.86 \pm 2.6$ & $24.49 \pm 3.0$ & $24.21 \pm 2.7$ & $25.46 \pm 2.8$ \\
\hline Height $(\mathrm{cm})$ & $155.76 \pm 8.9$ & $155.16 \pm 9.1$ & $155.24 \pm 9.4$ & $154.96 \pm 9.5$ \\
\hline Weight (kg) & $57.95 \pm 8.0$ & $59.09 \pm 9.2$ & $58.69 \pm 10.8$ & $61.48 \pm 11.1$ \\
\hline $\mathrm{SBP}(\mathrm{mmHg})$ & $129.4 \pm 14.2$ & $126.7 \pm 15.5$ & $130.6 \pm 13.1$ & $125.2 \pm 13.9$ \\
\hline $\mathrm{DBP}(\mathrm{mmHg})$ & $71.5 \pm 9.7$ & $68.8 \pm 10.6$ & $72.0 \pm 9.9$ & $68.3 \pm 10.4$ \\
\hline $\mathrm{FPG}(\mathrm{mg} / \mathrm{dL})$ & $169.25 \pm 22.8$ & $145.34 \pm 25.1^{\dagger}$ & $171.97 \pm 22.4$ & $144.1 \pm 25.1^{\dagger}$ \\
\hline $\mathrm{HbAlc}(\%)$ & $7.57 \pm 1.0$ & $6.96 \pm 1.0^{\dagger}$ & $7.69 \pm 0.9$ & $7.00 \pm 1.1^{\dagger}$ \\
\hline IRI $(\mu \mathrm{U} / \mathrm{mL})$ & $8.04 \pm 2.9$ & $7.33 \pm 3.7^{\dagger}$ & $7.88 \pm 3.0$ & $6.94 \pm 3.7^{\dagger}$ \\
\hline HOMA-IR & $3.31 \pm 0.4$ & $2.64 \pm 0.5^{\dagger}$ & $3.36 \pm 0.4$ & $2.47 \pm 0.4^{\dagger}$ \\
\hline $\mathrm{TC}(\mathrm{mg} / \mathrm{dL})$ & $218.17 \pm 27.2$ & $216.94 \pm 28.8$ & $215.84 \pm 27.4$ & $213.77 \pm 31.5$ \\
\hline $\mathrm{TG}(\mathrm{mg} / \mathrm{dL})$ & $177.57 \pm 38.3$ & $156.25 \pm 52.7^{*}$ & $172.32 \pm 37.9$ & $145.39 \pm 28.3^{\dagger}$ \\
\hline HDL (mg/dL) & $45.64 \pm 8.5$ & $49.17 \pm 8.2 *$ & $44.29 \pm 8.8$ & $48.84 \pm 8.8^{*}$ \\
\hline $\mathrm{LDL}(\mathrm{mg} / \mathrm{dL})$ & $137.02 \pm 16.2$ & $136.52 \pm 18.4$ & $137.08 \pm 17.2$ & $135.86 \pm 18.0$ \\
\hline
\end{tabular}

BMI, body mass index; SBP, systolic blood pressure; DBP, diastolic blood pressure; FPG, fasting plasma glucose; IRI, immunoreactive insulin; HOMA-IR, homeostasis model assessment for insulin resistance; TC, total cholesterol; TG, triglycerides; HDL, high density lipoprotein cholesterol; LDL, low density lipoprotein cholesterol.

$* p<0.05,{ }^{\dagger} p<0.01$ vs. baseline values. None of the intergroup differences reached statistical significance. 
doctor as to their general health, body weight, systolic and diastolic blood pressure (SBP and DBP), symptoms and signs suggestive of adverse reactions, and compliance with the medication, dietary therapy, and exercise therapy, at baseline and every month after the diagnosis during the 6-month treatment. BP was measured with the patient in sitting position after at least 5 minutes of rest.

We excluded subjects who had a history of glucoselowering pharmacotherapy or corticosteroids, and specific contraindications to pioglitazone including increased serum levels of liver enzyme (aspartate aminotransferase [AST] or alanine aminotransferase $[\mathrm{ALT}]>2.5$ times the upper limit of normal) and CHF defined as NYHA class III or IV. At study entry, antihypertensive agents except $\beta$-blockers and thiazides, and lipid-lowering agents, if administered for more than 3 months, were also allowed, provided that the dose was not changed during this study. None of the subjects was a heavy smoker or alcoholic.

The study was conducted in accordance with the Declaration of Helsinki, and the protocol was approved by the Ethical Committee of Rakuwakai Otowa Hospital. All the subjects gave their informed consent before they were enrolled.

\section{Biochemical measurements}

All subjects underwent laboratory blood tests at baseline, and every month after the diagnosis during the 6-month study period. Serum samples were obtained before 8:00 AM after an overnight fast, and were immediately processed and kept frozen at $-20^{\circ} \mathrm{C}$ until the assays were carried out. Serum AST, ALT, fasting plasma glucose (FPG), total cholesterol (TC), triglycerides (TG), and high density lipoprotein cholesterol (HDL) were measured using routine laboratory methods. Low density lipoprotein cholesterol (LDL) was calculated by Friedewald equation $(\mathrm{LDL}=\mathrm{TC}-$ $[\mathrm{HDL}+\mathrm{TG} / 5]) . \mathrm{HbA1c}$ was measured by the latex agglutination method (Fujirebio, Inc., Tokyo, Japan). Plasma insulin concentration (immunoreactive insulin [IRI]) was measured by enzyme immunoassay using a commercially available kit (Tosoh, Tokyo, Japan). The insulin resistance index was assessed by the homeostasis model assessment for insulin resistance (HOMA-IR).

\section{Statistical analysis}

Data were analyzed by the Student's unpaired t-test and paired t-test to assess intergroup differences and the longitudinal differences in each group, respective$1 \mathrm{y}$, and by $\chi^{2}$-test to assess intergroup differences of incidence. Statistics were calculated with Stat View version 5.0 (Abacus Concepts, Inc., Berkeley, CA). A $P$ value $<0.05$ was considered as statistically significant.

\section{Results}

Table 1 shows the baseline characteristics of patients in group A and group B. There was no significant difference between the two groups in terms of age, height, weight, body mass index (BMI), SBP, DBP, and biochemical parameters including TC, TG, HDL, FPG, $\mathrm{HbAlc}$, and IRI at baseline.

Mild peripheral edema occurred in 2 patients in group A and 11 patients in group B during this study. Among them 1 patient in group A and 10 in group B dropped out of the study. However, in these 13 patients, ultrasonography did not show any abnormalities, and peripheral edema was resolved spontaneously with cessation of pioglitazone. The incidence of peripheral edema was significantly much lower in group A than in group B $(\mathrm{p}=0.0014)$.

Body weight increased, but not significantly, from baseline during the treatment period in both groups (Table 1, Fig. 1). However, \% change of body weight in group A was significantly less than that in group B $(\mathrm{p}<0.0001)$ (Fig. 2).

Both SBP and DBP were lower than at baseline, but not significantly so, after treatment (Table 1). The \% change of BP was also not different between group A and group $\mathrm{B}$.

FPG, HbA1c, IRI, and HOMA-IR significantly improved from baseline during 6-month treatment in both groups (Table 1, Fig. 1). The $\%$ change of these parameters did not differ significantly between group A and group B (Fig. 2).

While TC and LDL hardly improved, TG and HDL significantly improved from baseline during 6-month treatment in both groups (Table 1, Fig. 1). The \% change of these parameters did not differ significantly between group A and group B (Fig. 2). 

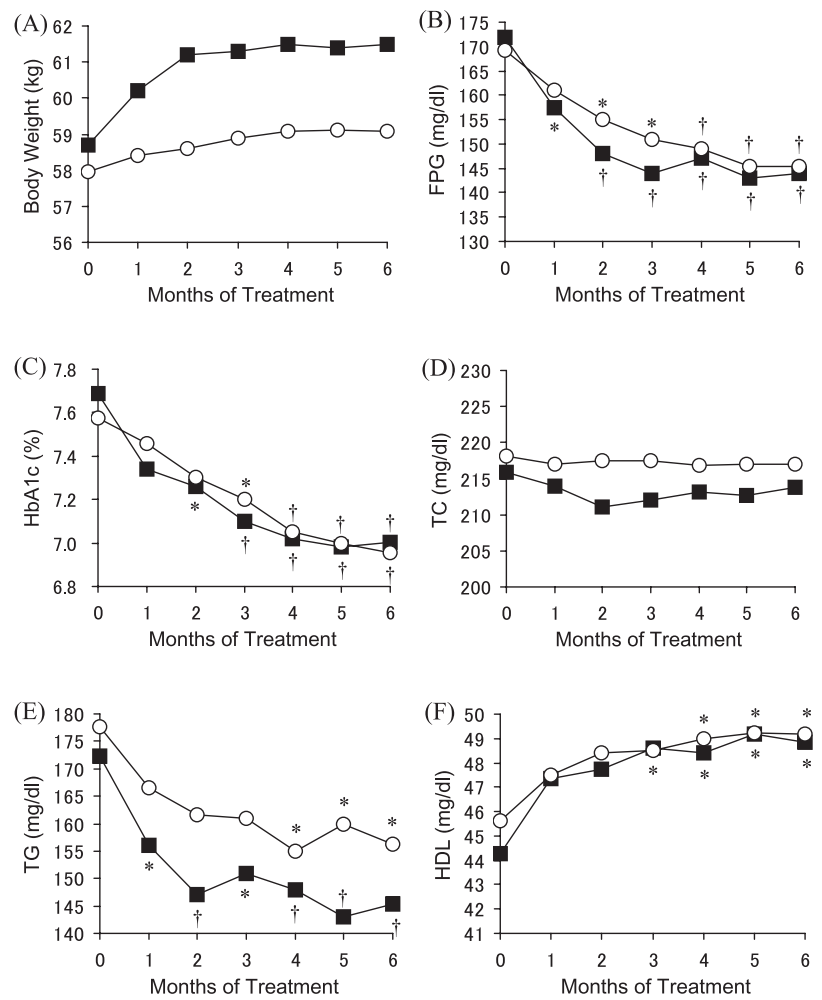

Fig. 1. Body weight (A), FPG (B), HbAlc (C), TC (D), TG (E), and HDL (F) (mean values) during the 6-month treatment in patients of group A (white circles) and group B (black squares). ${ }^{*} p<0.05,{ }^{\dagger} p<0.01$ vs. baseline values. None of the intergroup differences reached statistical significance.

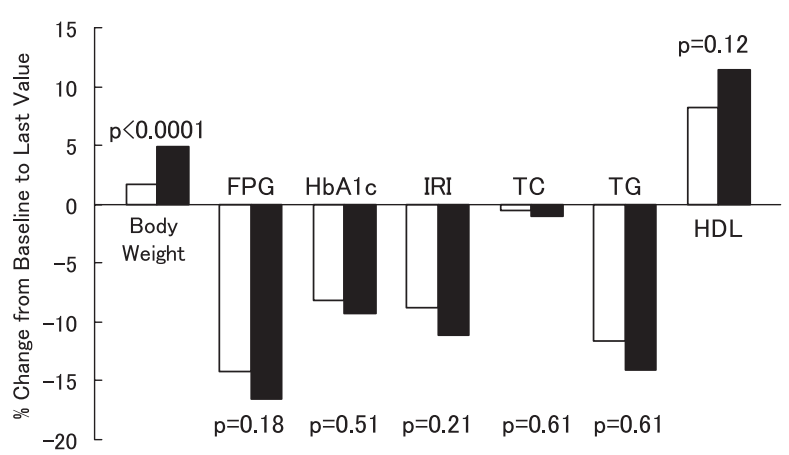

Fig. 2. Changes in body weight, FPG, HbA1c, IRI, and lipid profiles from baseline to last value in patients of group A (white bars) and group B (black bars). $P$-values for comparisons between group A and group B.

\section{Discussion}

In the present study of low-dose pioglitazone in Japanese women with T2DM, we found that low-dose pioglitazone ( $7.5 \mathrm{mg} /$ day) had a comparable benefit on glucose and lipid metabolism, compared with standarddose pioglitazone $(15 \mathrm{mg} /$ day). On the other hand, we also found that its deleterious effects, including weight gain and peripheral edema, were significantly more modest in low-dose pioglitazone. These results suggest that, considering its efficacy, safety, and costeffectiveness, low-dose pioglitazone may be preferred for treating Japanese women with T2DM compared with the standard-dose.

Previous studies in rats revealed that pioglitazone dose-dependently reduced hyperglycemia, hyperlipidemia, and hyperinsulinemia [16]. Since then, it has been clinically described that the effects of pioglitazone on glycemic control are dose-dependent $[3,4,10$, 14]. We do not deny these dose-dependent effects of pioglitazone, because responsiveness to pioglitazone was found to be relatively better in the $15-\mathrm{mg}$ group than in the $7.5-\mathrm{mg}$ group in our study, although not significantly so.

Recently, Aronoff et al. [10] evaluated the efficacy and safety of four doses of pioglitazone monotherapy in the treatment of patients with T2DM in a large-scale multicenter trial. While their results showed a dosedependent beneficial effect of pioglitazone on glycemic control in T2DM, its effect on glycemic control was comparable between the $15-\mathrm{mg}$ group and the $30-\mathrm{mg}$ group. The baseline characteristics in their study were somewhat different from those in ours. While all the patients in our study are Japanese and female, and had never received pharmacological antidiabetic therapy, those in their study were racially-mixed, included both men and women, and only $31 \%$ of them were naive to prior antidiabetic therapy. Another difference is that the baseline $\mathrm{HbA1c}$ and FPG were relatively higher in their study $(>10.0 \%$ and $>260 \mathrm{mg} / \mathrm{dL}$, respectively) than in our study. It has been suggested in the literature that the magnitude of the response to pioglitazone should be greater in those naive to prior antidiabetic therapy and in those with better metabolic control [4, 5], as Aronoff et al. stated in their report [10]. Therefore, the response to low dose pioglitazone may have been greater in our study due to earlier diagnosis or better metabolic control at the onset.

More recently, Miyazaki et al. [14] also investigated the dose-response effects of pioglitazone on glycemic control in patients with T2DM. While the dosedependent beneficial effects of pioglitazone on glycemic control were shown also in their study, the improve- 
ment of FPG in the 7.5-mg group was superior to that in the 15-mg group. Furthermore, considering that the baseline $\mathrm{HbAlc}$ in their study had been substantially higher in the 7.5-mg group than in the $15-\mathrm{mg}$ group, it can be said that the 15-mg group had a potential advantage over the 7.5-mg group in responding to pioglitazone and, therefore, that the improvement of $\mathrm{HbAlc}$ was comparable between the 7.5- and 15-mg group in their study. Thus, their results are supportive of ours which showed comparable effects on glycemic control between the 7.5-mg group and the 15-mg group.

In the Japanese literature, there have been several studies showing that the effect of $15-\mathrm{mg}$ pioglitazone administered every other day on glycemic control is comparable with that of 15 -mg pioglitazone everyday in patients with T2DM [17-19]. Another study described that reducing dose of pioglitazone from $15 \mathrm{mg} /$ day to $7.5 \mathrm{mg}$ /day did not alter metabolic control in Japanese patients with T2DM ( 3 men and 8 women) [20]. These findings are compatible with our results, indicating that $7.5-\mathrm{mg}$ pioglitazone is potentially useful in improving glycemic control.

It has been proposed that TZDs including pioglitazone may correct other metabolic abnormalities, such as dyslipidemia and arterial hypertension $[1-6,10,14$, $15,21-24]$, as found also in our study. In a study examining Japanese patients with T2DM, a statistically significant increase of HDL from the baseline was found with 7.5-mg pioglitazone [25], consistent with our results. These beneficial effects of pioglitazone are considered to be mainly attributed to the improvement of insulin resistance [1-6]. In the study by Miyazaki et al. [13] reduction in IRI was comparable among the subgroups treated with different doses of pioglitazone, and 7.5-mg pioglitazone substantially decreased IRI (means \pm SEM) from $25 \pm 5 \mu \mathrm{U} / \mathrm{mL}$ to $20 \pm 3 \mu \mathrm{U} / \mathrm{mL}$ during the 26-week treatment period. Their findings were consistent with ours, suggesting the beneficial effects of 7.5-mg pioglitazone.

The severity of weight gain and peripheral edema due to pioglitazone has also been reported to be dosedependent $[4,10,14]$, as seen in our study. A considerably high percentage $(26.8 \%)$ of our patients treated with 15-mg pioglitazone did experience edema, although mild, and they refused to take pioglitazone any longer even if its dose was reduced. However, weight gain and edema were almost negligible in our patients treated with $7.5-\mathrm{mg}$ pioglitazone, suggesting the safety of $7.5 \mathrm{-mg}$ pioglitazone. Additionally, consistent with previous reports $[2-6,10,14,15]$, there were no cases of drug-induced hepatotoxicity or hypoglycemia in either group in our study, while hypoglycemia is a common adverse event in other glucose-lowering agents [26].

In conclusion, we showed that $7.5-\mathrm{mg}$ pioglitazone significantly improved glucose and lipid metabolism, and, in addition, it was well tolerated in this study. Our results demonstrate the safety and efficacy of $7.5-\mathrm{mg}$ pioglitazone per day, suggesting that low-dose pioglitazone $(7.5 \mathrm{mg}$ /day) could be another good choice of treatment for Japanese women with T2DM.

\section{Acknowledgements}

We wish to thank Norihito Izumi (Takeda Pharmaceutical Company, Ltd., Kyoto) for providing information on pioglitazone.

\section{Reference}

1. Inzucchi SE, Maggs D, Spollet GR, Page SL, Rife FS, Walton V, Shulman GI (1998) Efficacy and metabolic effects of metformin and troglitazone in type II diabetes mellitus. N Engl J Med 338: 867-872.

2. Parulkar AA, Pendergrass ML, Granda-Ayala R, Lee TR, Fonseca VA (2001) Nonhypoglycemic effects of thiazolidinediones. Ann Intern Med 134: 61-71.

3. Stumvoll M, Haring HU (2002) Glitazones: clinical effects and molecular mechanisms. Ann Med 34: 217224.

4. O'Moore-Sullivan TM, Prins JB (2002) Thiazolidinediones and type 2 diabetes: new drugs for an old disease.
Med J Aust 176: 381-386.

5. Edelman SV (2003) The role of the thiazolidinediones in the practical management of patients with type 2 diabetes and cardiovascular risk factors. Rev Cardiovasc Med 4: S29-37.

6. Kawamori R, Kadowaki T, Onji M, Seino Y, Akanuma Y (2004) Internal analysis on PRACTICAL (Prospective Actos practical experience) Study; Efficacy and Safety in over 20,000 Japanese Type 2 Diabetic Patients. Diabetes 53 (S-2): A156.

7. Satoh N, Ogawa Y, Usui T, Tagami T, Kono S, Uesugi H, Sugiyama H, Sugawara A, Yamada K, Shimatsu A, 
Kuzuya H, Nakao K (2003) Antiatherogenic effect of pioglitazone in type 2 diabetic patients irrespective of the responsiveness to its antidiabetic effect. Diabetes Care 26: 2493-2499.

8. Maeda K (2001) Hepatocellular injury in a patient receiving pioglitazone [letter]. Ann Intern Med 135: 306.

9. Nesto RW, Bell D, Bonow RO, Fonseca V, Grundy SM, Horton ES, Le Winter M, Porte D, Semenkovich CF, Smith S, Young LH, Kahn R (2004) Thiazolidinedione use, fluid retention, and congestive heart failure: a consensus statement from the American Heart Association and American Diabetes Association. Diabetes Care 27: 256-263.

10. Aronoff S, Rosenblatt S, Braithwaite S, Egan JW, Mathisen AL, Schneider RL (2000) Pioglitazone hydrochloride monotherapy improves glycemic control in the treatment of patients with type 2 diabetes. Diabetes Care 23: 1605-1611.

11. Mathisen A, Geerlof J, Houser V (1999) The effect of pioglitazone on glucose control and lipid profile in patients with type 2 diabetes. Diabetes 48: A102-A103.

12. Gillies PS, Dunn CJ (2000) Pioglitazone. Drugs 60: 333-343.

13. Rubin C, Egan J, Schneider R (1999) Combination therapy with pioglitazone and insulin in patients with type 2 diabetes. Diabetes 48: A110.

14. Miyazaki Y, Matsuda M, DeFronzo RA (2002) Doseresponse effect of pioglitazone on insulin sensitivity and insulin secretion in type 2 diabetes. Diabetes Care 2002 25: 517-523.

15. Schernthaner G, Matthews DR, Charbonnel B, Hanefeld M, Brunetti P (2004) Quartet [corrected] Study Group. Efficacy and safety of pioglitazone versus metformin in patients with type 2 diabetes mellitus: a double-blind, randomized trial. J Clin Endocrinol Metab 2004 89: 6068-6076.

16. Sugiyama Y, Taketomi S, Shimura Y, Ikeda H, Fujita $\mathrm{T}$ (1990) Effects of pioglitazone on glucose and lipid metabolism in Wistar fatty rats. Arzneimittelforschung
40: $263-267$.

17. Koyama K (2003) Comparison of availability and safety when administered pioglitazone $15 \mathrm{mg}$ every one day or two days to type 2 diabetic patients. Prog Med 23: 2417-2421 (Abstract in English).

18. Taneda Y, Ono T, Shiga N, Umemura S (2005) Efficacy of 15-mg pioglitazone administered every other day. J Jpn Diab Soc 48: 209 (in Japanese).

19. Watabe R, Kamimoto M (2005) Efficacy of 15-mg pioglitazone administered everyday and every other day. J Jpn Diab Soc 48: 209 (in Japanese).

20. Okada Y, Iguchi N, Kishikawa H, Hirose A, Kanda K, Morita E, Tanaka Y (2005) Efficacy and safety of lowdose pioglitazone (7.5 mg/day). Diabetes Frontier 16: 123-126 (in Japanese).

21. Buchanan TA, Meehan WP, Jeng YY, Yang D, Chan TM, Nadler JL, Scott S, Rude RK, Hsueh WA (1995) Blood pressure lowering by pioglitazone. Evidence for a direct vascular effect. J Clin Invest 96: 354-360.

22. King AB (2000) A comparison in a clinical setting of the efficacy and side effects of three thiazolidinediones. Diabetes Care 23: 557.

23. Orbay E, Sargin M, Sargin H, Gozu H, Bayramicli OU, Yayla A (2004) Addition of rosiglitazone to glimepirid and metformin combination therapy in type 2 diabetes. Endocr J 51: 521-527.

24. Dereli D, Dereli T, Bayraktar F, Ozgen AG, Yilmaz C (2005) Endocrine and metabolic effects of rosiglitazone in non-obese women with polycystic ovary disease. Endocr J 52: 299-308.

25. Kaneko T, Baba S, Toyota T, Akanuma Y, Sakamoto N, Shigeta Y, Shichiri M (1997) Efficacy of pioglitazone (AD-4833) in patients with NIDDM treated only with diet therapy. Jpn J Clini Exper Med 74: 12271249 (in Japanese).

26. Miwa S, Watada H, Ohmura C, Tanaka Y, Kawamori R (2004) Efficacy and safety of once daily gliclazide (20 mg/day) compared with nateglinide. Endocr $J$ 51: 393-398. 